\title{
QUALITY PARAMETER ASSESSMENT ON IRIS IMAGES
}

\author{
October 8, 2014 \\ CSIR, Modeling and Digital Science, \\ P O Box 395, Pretoria, South Africa
}

Sisanda Makinana, Tendani Malumedzha and Fulufhelo V. Nelwamondo

\begin{abstract}
Iris biometric for personal identification is based on capturing an eye image and obtaining features that will help in identifying a human being. However, captured images may not be of good quality due to variety of reasons e.g. occlusion, blurred images etc. Thus, it is important to assess image quality before applying feature extraction algorithm in order to avoid insufficient results. Poor quality images may affect the recognition as they have few sufficient feature information. Moreover, existing quality measures focuses on parameters or factors than feature information. In this paper, iris quality assessment research is extended by analysing the effect of entropy, contrast, area ratio, occlusion, blur, dilation and sharpness of an iris image which determines the iris size, amount of information and clearness of the features. A weighting method based on principal component analysis (PCA) is proposed to determine the influence each parameter has on the quality score. To test the proposed technique; Chinese Academy of Science Institute of Automation (CASIA), Internal Collection (IC) and University of Beira Interior (UBIRIS) databases are used. A conclusion is drawn that the combination of blur, dilation and sharpness parameters have the most influence in the quality of the image as they weighed more than other parameters
\end{abstract}

\section{Introduction}

Iris recognition is an automated method of biometric identification that analyses patterns of the iris to identify an individual [1]. It is said to have high reliability in identification because each individual has unique iris patterns [2], [3]. However, due to the limited effectiveness of imaging, it is important that image of high-quality are selected in order to improve iris recognition performance. Some advanced preprocessing algorithms can process poor quality images and produce adequate results, however they are computationally expensive and add extra burden on the recognition system time. Therefore quality determination is necessary in order to determine which algorithm to use for preprocessing. For example, if it is known that the acquired image does not meet the desired quality it can be subjected to stricter preprocessing algorithms selectively. Various quality assessment methods have been developed to ensure quality of the sample acquisition process for online systems [4]. These approaches are good for quick elimination of poor quality images and even images from which an accurate segmentation may be produced are eliminated. A more discriminative approach to quality, images can be assigned quality levels, which will provide an indication as to whether further processing can enhance them.

In this paper, the focus is on character component of a biometric sample quality due to the observation that available algorithms utilise and focus on fidelity and utility components [4], [5], [6]. There are various quality parameters that may affect iris images; these include defocus, motion blur, entropy, contrast, occlusion and dilation. Several authors have proposed algorithms that address individually the following parameters: defocus blur, 
motion blur and occlusion[6] or pair them with other quality parameters [4], [5]. A complete assessment of iris image quality is still a challenge because the overall iris image quality score is determined by multiple quality parameters and there is currently no well-defined standard for determining the weight of each individual quality parameter.

This paper proposes an algorithm that assesses the quality of an iris image based on multiple quality parameters. Firstly, image quality parameters are estimated, i.e. mean contrast, sharpness, blur, dilation, area ratio, entropy and occlusion. Thereafter, a fusion technique based on principal component analysis is used to weight each quality parameter and obtain a quality score for each image. The remainder of this paper is organised as follows. Section 2 describes the overview of the proposed method. Section 3 provides the estimations of individual quality parameters and discusses the implementation of the proposed fusion method. Last sections provides experimental results and a conclusion.

\section{Iris image quality overview}

The approach taken in this paper is to estimate individual quality parameters and fuse them using principal component analysis to obtain a quality score. Fig. 1 illustrates the overview of the iris assessment plan.

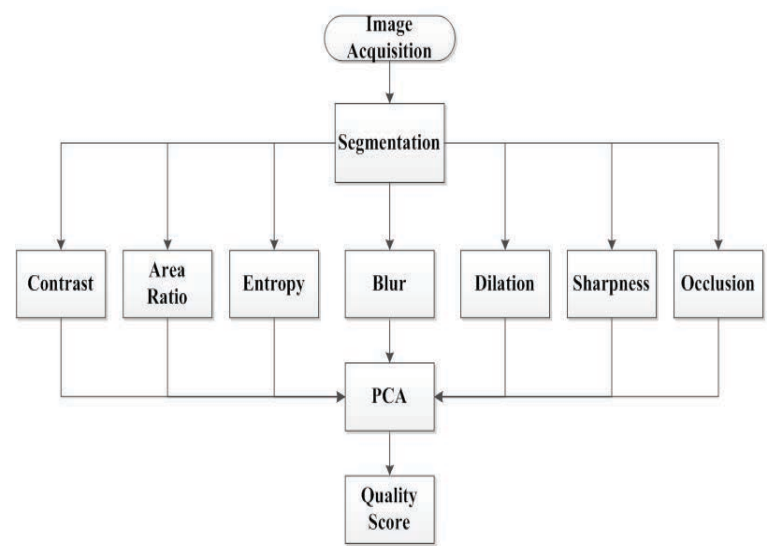

Figure 1. The framework of the proposed scheme

\section{Estimation of quality parameters}

The proposed algorithm for quality assessment is intended to be used during the acquisition pro- cess. The objective is to assess the defined quality parameters and determine which quality measure mostly affect the quality score. Implementation of assessment algorithm is carried out two steps: namely, segmentation of iris samples and estimation of quality parameters. The subsections below details how this is done.

\subsection{Segmentation}

This stage involves segmenting the iris image, which is carried out by invoking Masek's [2] publicly available automatic segmentation method. It is based on the Hough transform, and is able to localise the circular iris and pupil region, occluding iris-lids, iris-lashes and reflections. Hough Transform is an extraction technique that uses the radius values to detect circles in an image. The circular Hough transform was used for detecting the iris and pupil boundaries. This involves first employing canny edge detection to generate an edge map. The Canny edge detector is an operator that uses a multi-stage algorithm to detect a wide range of edges in images. The range of radius values was set manually, depending on the database used. For CASIA and IC databases the values of the iris radius range from 80 to 150 pixels, while the pupil radius ranges from 28 to 75 pixels. For UBIRIS database the range for pupil radius is 5 to 11 pixels and for the iris radius it ranges from 39 to 49 pixels. The Hough transform determines the iris boundary first, and then determines the pupil boundary within the iris region. After getting the iris and pupil boundaries, the iris and pupil image is displayed with everything beyond the iris radius masked out. Fig. 2 illustrates the segmented image of CASIA.

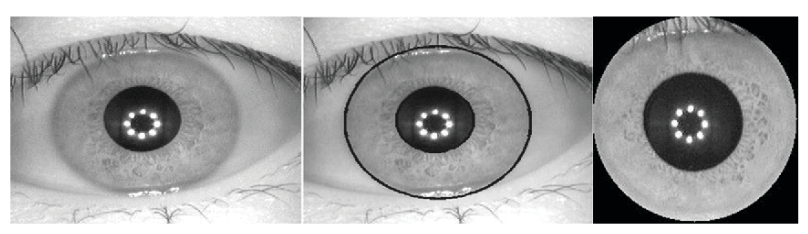

Figure 2. The framework of the proposed scheme 


\subsection{Estimation of predefined quality pa- rameters}

The following are the quality parameters that are estimated for the proposed algorithm:

\subsubsection{Contrast}

The term contrast refers to the representation of colour and difference in luminance that makes a feature noticeable within an image [7]. However human vision is more sensitive to difference in colour representation than difference in luminance. According to human visual, contrast is the difference in colour and brightness of various objects within the same filed of view.

Contrast determines the clearness of the features within an image. High contrast means the more clearer the iris features and making easier for feature extraction. Assessing contrast is important to ensure sufficient and clear features are extracted. In measuring contrast a window of $8 \times 8$ pixels is defined, of which the Fast Fourier Transform (FFT) of the 2-Dimensional image is computed for each sub-window. FFT transforms the signal from time domain into a frequency domain and is defined as [8]

$$
\begin{gathered}
H(u, v)= \\
=\sum_{x=0}^{M-1} \sum_{y=0}^{M-1}\left(\exp \left[-2 \pi i y \frac{v}{M}\right] \exp \left[-2 \pi i x \frac{u}{M}\right] h(x, y)\right)
\end{gathered}
$$

where $\mathrm{u}, \mathrm{v}=-\frac{M}{2} \ldots \cdot \frac{M}{2}$. The squared magnitude of the Fourier Series coefficients which indicates power at corresponding frequencies is computed by Parseval's Theorem [9]

$$
P(u, u)=|H(u, v)|^{2}=\operatorname{Re}(u, v)+\operatorname{Im}(u, v)^{2}
$$

The fundamental frequency (dcpower), the total power (totpower) and the non-zero power (acpower) of the spectrum is computed which are [9]

$$
\begin{gathered}
\text { dcpower }=P(0,0)=|H(0,0)|^{2} \\
\text { avgGray }=\frac{(\text { dcpower })^{\frac{1}{2}}}{M^{2}} \\
S_{k}=\sqrt{\frac{1}{N^{2}} \sum_{x=1}^{N} \sum_{y=1}^{N}\left(I_{x y}-I_{k}\right)^{2}} \\
S_{S T D}=\frac{1}{N} \sum_{k=1}^{N} S_{k}
\end{gathered}
$$

In equation $5, I_{x, y}$ is the gray level of pixel $(x, y)$ and $I_{k}$ is the average gray level of the $k^{t h}$ region. The average intensity or contrast is computed as follows

$$
M_{C}=\frac{S_{S T D}}{\operatorname{avgGray}}
$$

\subsubsection{Area Ratio}

The representation of pattern recognition should be invariant to the change in size, position and orientation of the iris image. If the captured iris was far from the capturing device, the image might be small, which means fewer features will be resolvable for extraction. Also, if the subject is too close to the capturing device that may cause the captured image to be blurry. Thus, it is of utmost importance to assess the iris area ratio; which is defined as:

$$
M_{A}=\frac{A_{I}}{A_{E}}
$$

where $A_{I}$ is the area of the iris and $A_{E}$ is the area of the entire image.

\subsubsection{Entropy}

Entropy is a statistical measure of randomness that can be used to characterize the texture of the input image. It is the quantity of information contained in the image. It's also given as the uncertainty associated with random variables. Entropy is defined as

$$
M_{E}=-\sum_{i=0}^{255} p_{i} \log p_{i}
$$

where

$$
p_{i}=\frac{N_{i}}{N}
$$

In $10 N_{i}$ is the number of pixels with grey level and $\mathrm{N}$ is the total number of pixels in the image. $p_{i}$ is the probability of occurrence of grey level intensities.

\subsubsection{Blur}

Blur may result from many sources, but in general it occurs when the focal point is outside the depth of field of the captured object. The further the object is from the focal point of the capturing device, the higher degree of blur in the output image [6]. On the databases used there is no significant blur, so a low-pass Gaussian filter was convolved to 
the iris input image in order to estimate blur. First, the intensity variations between neighbouring pixels of the input image and blurred image are computed. Then, the intensity variations of these two images are compared; the results obtained are used as the estimation of blur. This blur estimation is based on Crete et al [10] approach. The blur estimation procedure is illustrated in Fig. 3.

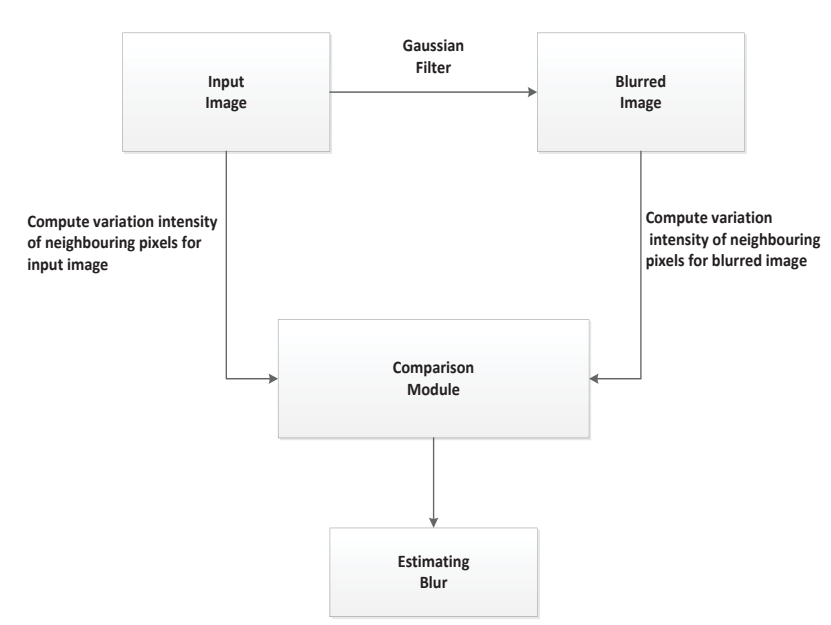

Figure 3. The flowchart of the blur estimation [10]

\subsubsection{Dilation}

The variation in pupil dilation between the enrolment image and the image to be recognised or verified may affect the accuracy of iris recognition system. A degree of dilation was measured for each iris image. The segmentation results provided the radius of the pupil and of the iris. To measure dilation, a ratio of radius of pupil to radius of iris was calculated. Since the pupil radius is always less than the radius of iris, the ratio will fall between 0 and 1 . The dilation measure $M_{D}$ is calculated by

$$
M_{D}=\frac{P_{R}}{I_{R}}
$$

where $P_{R}$ is the pupil radius and $I_{R}$ is the iris radius.

\subsubsection{Sharpness}

Images are usually affected by distortions during acquisition and processing, which may result in loss of visual quality. Therefore, image focus assessment is useful in such applications. Sharpness is arguably the most important quality parameter because it determines the amount of readable information an image may hold. Sharpness generally attenuates high frequencies. Due to that factor, sharpness can be assessed by measuring high frequencies in the image. Daugman illustrated this in [4] by proposing a ( 8 X 8) convolution kernel. To assess the appearance of the blur effect, a sharpness metric is proposed in the literature. Sharpness is estimated based on the gradient of the image to determine whether the image is in focus or not, because the gradient of an image is the directional change in the intensity of an image. The gradient of the image is given by:

$$
\nabla G=\left(\frac{\partial G}{\partial x} \hat{x}\right)+\left(\frac{\partial G}{\partial y} \hat{y}\right)
$$

where $\frac{\partial G}{\partial x}$ and $\frac{\partial G}{\partial y}$ are the gradients in the $\mathrm{x}$ and $\mathrm{y}$ direction respectively.

The sharpness $\left(M_{S}\right)$ is calculated by dividing the sum of gradient amplitude (S) by the number of elements of the gradient $(\mathrm{T})$.

$$
M_{S}=\sum(S) / T
$$

The gradient amplitude (S) is given by

$$
S=\sqrt{G_{x}^{2}+G_{y}^{2}}
$$

where $G_{x}$ and $G_{y}$ in 14 are the horizontal and vertical change in intensity.

\subsubsection{Occlusion}

The occlusion measure $\left(M_{O c c}\right)$ is the amount of iris region that is invalid due to obstruction by eyelids and eyelashes. Eyelid and eyelashes occlusion problem is a primary cause of bad quality in iris image [11]. Compared with the edge of iris texture, the edge of iris-lid and iris-lash is much sharper and usually considered to contain high pixel values. To estimate the amount of occlusion at each level an occlusion is measured by calculating the total grey value of the image[12]. It is defined as

$$
M_{O c c}=\frac{1}{A_{I} \times A_{E}}
$$

where $A_{I}$ and $A_{E}$ in are the area of the iris and Area of iris-lids. The higher the metric value the greater is the chance for occlusion by iris lid. 


\section{Fusion Technique}

A unique quality score is of value to the prediction step of iris recognition system. To obtain this quality score, a fusion technique based on Principal Component Analysis (PCA) is proposed. PCA is a widely used tool which is proficient in reducing dimensions and determining factor relationships amongst datasets just like Factor Analysis (FA) [13]. However, FA evaluates the linear relationship between the number of variables of interest $Y_{1}, Y_{2}, \ldots . Y_{j}$, and a smaller number of unobserved factors $F_{1}, F_{2}, \ldots, F_{k}$, whereas, PCA is a technique that determines the factor loadings of the dataset by calculating the eigenvectors and eigenvalues of the covariance matrix [14]. In this research PCA has been used over FA since the interest is in determining the factor loading of the dataset. Factor loadings are the weights of each variable and correlations between each factor [15].

The PCA is calculated by defining the eigenvectors and eigenvalues of the covariance matrix. The covariance matrix measures the variation of the dimensions from the mean with respect to each other. Prior to applying the PCA, quality parameters need to be normalised. The quality parameters is standardized using the $Z_{s}$ before obtaining the first PCA, which is

$$
Z_{s}=\frac{x-\mu}{\sigma}
$$

where $\mu$ is the mean and $\sigma$ is the standard deviation of the estimated measures of the entire database.

Suppose $n$ independent observation are given on $X_{1}, X_{2}, \ldots, X_{k}$, where the covariance $X_{i}$ and $X_{j}$ is

$$
\operatorname{Cov}\left(X_{i}, X_{j}\right)=\sum i, j
$$

for $i, j=1,2, \ldots, k$ in equation 17 . Then the eigenvalues and eigenvectors of the covariance matrix are calculated. Since the observations are normalised then

$$
v_{j}^{T} v_{j}=1
$$

where $v$ is the eigenvectors of the covariance matrix. Then $W$ is defined to be the first principal component. It is the linear combination of the $X^{\prime} s$ with the largest variance:

$$
W=a_{1}^{T} X_{i}
$$

where $i=1,2, \ldots k$ and $a$ is the eigenvector of the covariance which also implies that $a_{j}^{T} a_{j}=1$. Then the quality score is obtained by multiplying normalised measures of parameters with weights for each quality parameter of the image. The fusion quality index is given as

$$
Q_{s}=\sum_{i=1}^{N} Q_{p} W_{p}
$$

where $Q_{s}$ is the quality score, $Q_{p}$ is the estimated quality parameter and $W_{p}$ is the amount of influence each parameter has on the quality score. The scores represent the global quality score of the iris segmented images.

\section{Dataset Used For Analysis}

In this paper, the CASIA and UBIRIS databases which are available free online and Internal Collection (IC), database were used, to estimate the quality parameters and their scores. For CASIA a subset of images called 'interval' was used. It contained 525 images which were captured at a resolution of $320 \times 280$ pixels. UBIRIS consists of 917 images captured at a resolution of $200 \times 150$ pixels. IC consists of 116 images captured at a resolution of 640 x 480 pixels.

\section{Image Description}

For UBIRIS database images were captured on two different sessions. For the first session noise factors like reflection, luminosity and contrast were minimized by capturing images inside a dark room. In the second session capturing location was changed to introduce noisy images. This introduced diverse images with respect to reflection, contrast, focus and luminosity problems [16].

For CASIA database images were captured by a closed up iris camera with circular NIR LightEmitting Diode (LED) array which had suitable luminous flux for iris imaging. The camera captures very clear iris images [17].

The IC iris database which was also used for testing the algorithm is a new database its images were collected in the Council of Scientific and Industrial Research (CSIR) campus in Pretoria. A Vista EY2 iris camera was used to collect these images. 


\section{Quality Parameter Distribution}

This section discusses the distribution of each quality parameter, also the histograms of the estimated quality parameters for UBIRIS, IC and CASIA databases are presented. The $x$-axis for all plots represents the strength of the estimated parameters.

Fig. 4 shows a histogram plots of contrast estimates for all the databases. All three databases have a wide range of contrast scores ranging above 0.5 , with UBIRIS database having the highest contrast scores for all the databases. The reason for high scores of UBIRIS is because images were captured in a controlled room where noise factors relative to reflection, luminosity and contrast were minimized. Image acquisition for this database was done in a dark room to eliminate ambient light which has a negative effect on contrast of the image.

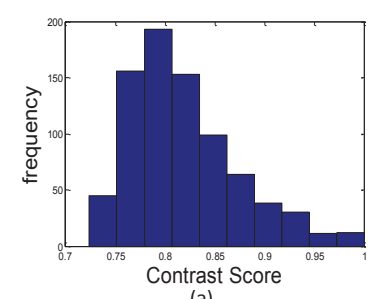

(a)

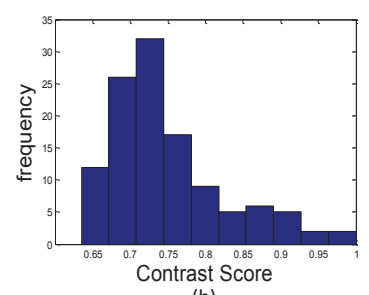

(b)

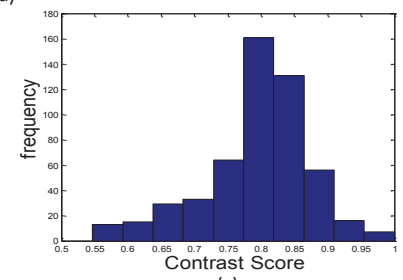

(c)

Figure 4. Contrast Distribution of (a) UBIRIS, (b) IC and (c) CASIA Databases respectively

Fig. 5 shows histogram plots of entropy estimates. For all three databases, there are high values of entropy. All three database have high mean values and moderate values of standard deviation meaning that the quality value do not deviate from the mean. The results here indicate significant contrast values for each database as contrast determines how clear the details of the images are.

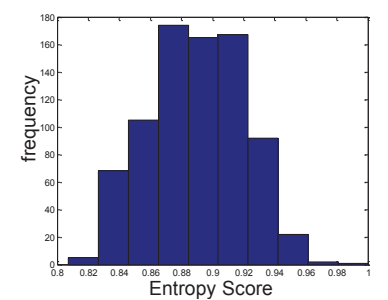

(a)

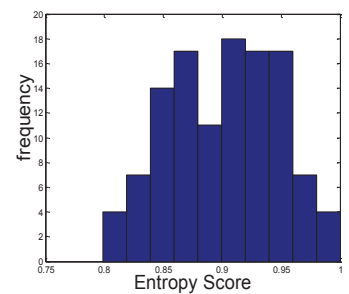

(b)

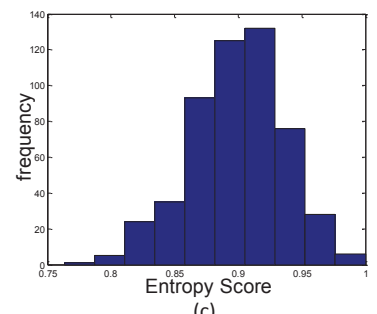

Figure 5. Entropy Distribution of (a) UBIRIS, (b)

IC and (c) CASIA Databases respectively

Fig. 6 shows histogram plots of sharpness estimates. All three databases are negatively influenced by sharpness scores, with UBIRIS being affected more by sharpness than the other databases. The main cause of this negative influence is the external environmental conditions which the images were captured in. The image capturing process for UBIRIS was done in two sessions. One session images were captured in a location that had natural luminosity which caused images to have contrast, luminosity and focus problems. These problems caused most images to have low scores of sharpness. In the other session images were captured in a controlled environment where noise relative to luminosity, contrast and reflection was minimized. Images captured in this session have high sharpness values. For the IC database, images were captured in an uncontrolled environment which caused a high amount of images to have low scores of sharpness. However there are some images with high sharpness which were captured in the afternoon. 


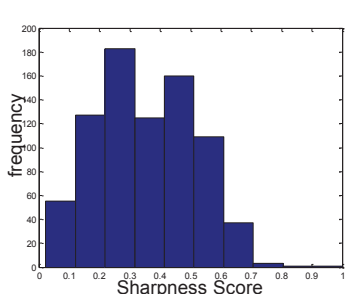

(a)

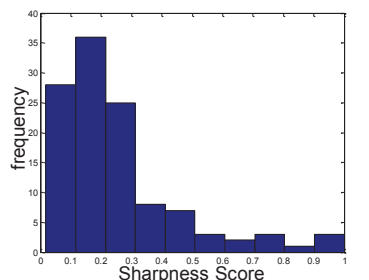

(b)

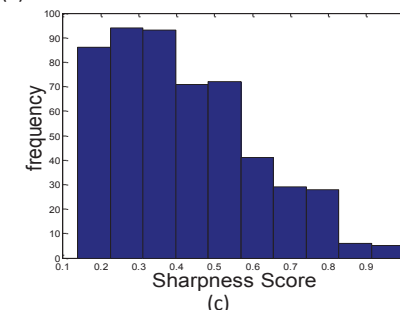

(c)

Figure 6. Sharpness Distribution of (a) UBIRIS, (b) IC and (c) CASIA Databases respectively

Fig. 7 illustrates the histogram plots of occlusion estimates. For all databases the amount of occlusion is sensible, however the CASIA database contains the highest occlusion scores, with mean of 0.2948 and standard deviation of 0.2140 . For UBIRIS there are many images with occlusion scores ranging from 0.1 to 0.5 meaning there is significant evidence that this database is not highly affected by occlusion. The results presented here imply that during iris acquisition most individuals eyelashes did not occlude their iris. For the IC database, individuals were instructed to open their eyes widely to eliminate occlusion during iris acquisition. However some iris images were occluded due to the fact that some individuals have small eyes and long eyelashes. Their images acquired during data collection were either significantly good or highly occluded. This resulted in very few iris images being highly occluded with their values ranging from 0.9 to 1 . For CASIA it is visually clear that most individuals had long eyelashes which caused the database to contain the highest occlusion scores for the three databases.

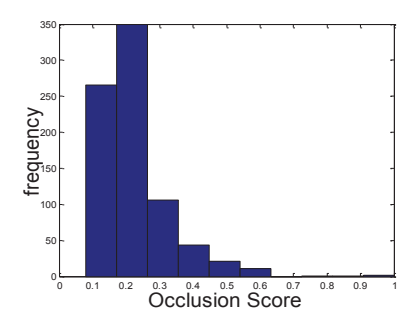

(a)

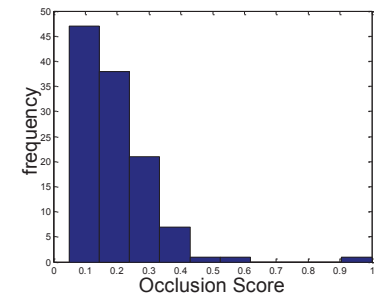

(b)

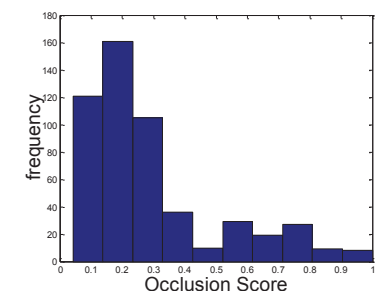

(c)

Figure 7. Occlusion Distribution of (a) UBIRIS, (b) IC and (c) CASIA Databases respectively

Fig. 8 shows the histogram plots of the area ratio estimates. The histograms shows that none of these databases are negatively influenced by this parameter as most images have an adequate score of area ratio ranging from 0.8 to 1 . In rating the databases UBIRIS has the highest amount of images having high area ratio scores followed by the IC database and then the CASIA database with some amount of images with low area ratio score which is caused by occlusion and failed segmentation. For all databases there are images having low scores of area ratio ranging from 0 to 0.2 . This is due to failed segmentation and pupil dilation which resulted in very low score of area ratio. An example of these images is illustrated in Fig. 9, 10 and 11. UBIRIS database contains a high amount of images with sufficient area ratio. As the scores increase from 0.4 to 1 the frequency also increases. These results indicates that the UBIRIS database contains iris images with very small iris size and also images of sufficient iris size. This also applies to the IC and CASIA databases, however, most images for these databases have area ratio scores between 0.8 and 0.9 . 


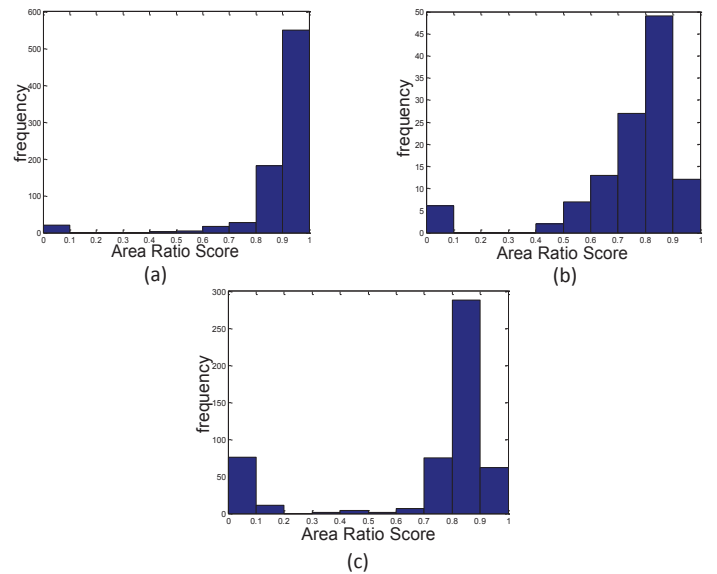

Figure 8. Area Ratio Distribution of (a) UBIRIS, (b) IC and (c) CASIA Databases respectively
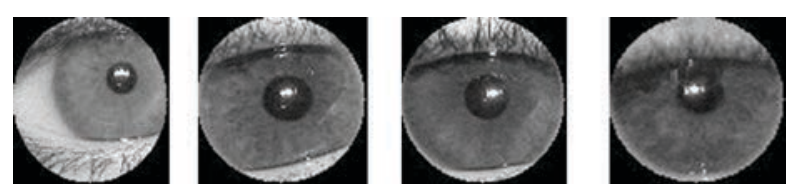

Figure 9. Failed segmentation for UBIRIS database
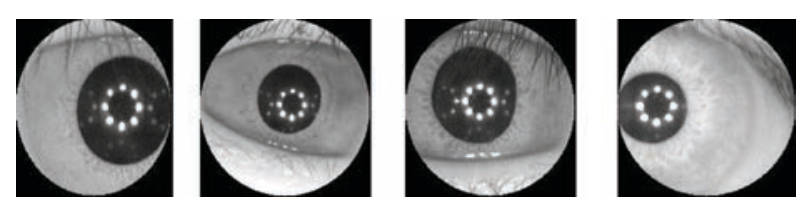

Figure 10. Failed segmentation for CASIA database
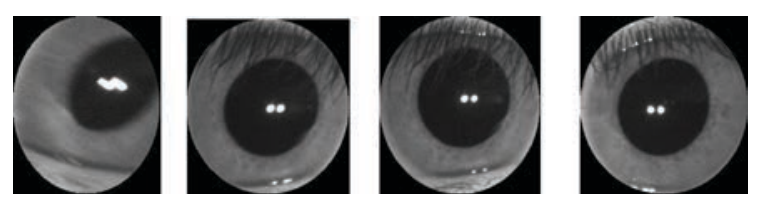

Figure 11. Failed segmentation and dilated iris images for IC database

Fig. 12 shows the histogram plots of dilation estimates. UBIRIS has the highest amount of images which are dilated compared to the other databases, with mean of 0.5462 and standard deviation of 0.0323 , which implies that an average of over $50 \%$ are images that are dilated in this database. On the other hand CASIA has the lowest values of dilation scores, with mean of 0.4516 and standard deviation of 0.1124 .
Image capturing in the dark causes the pupil to dilate and with light the pupil contracts. As stated in section 6 some iris images of UBIRIS and IC were captured in the dark causing high amount of iris images with pupil dilation scores ranging from 0.4 to 0.75 .

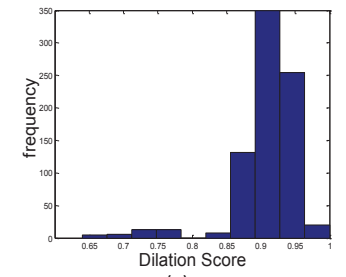

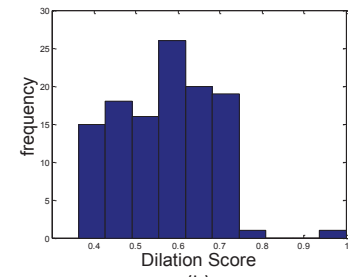

(b)

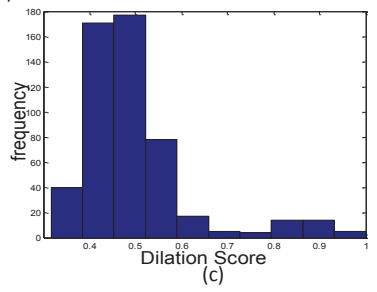

Figure 12. Dilation Distribution of (a) UBIRIS, (b) IC and (c) CASIA Databases respectively
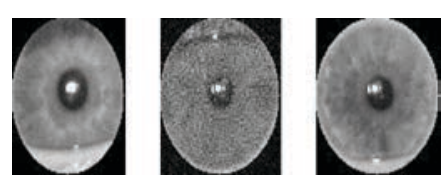

Figure 13. An example of blurred images of UBIRIS Database

Fig. 14 shows the histogram of blur estimates. IC and UBIRIS databases are more affected by this parameter. During iris capturing for UBIRIS database the environment introduced diverse imaging problems with respect to reflection, contrast, focus and luminosity which caused the high blur scores. It is also visually clear that UBIRIS database is affected by blur as shown in fig. 13. 
(a)

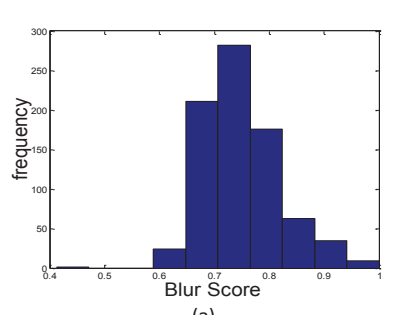

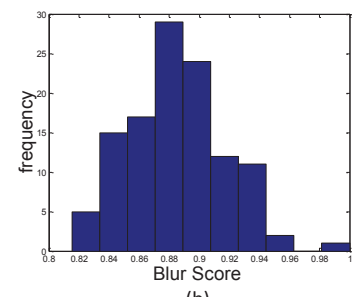

(b)

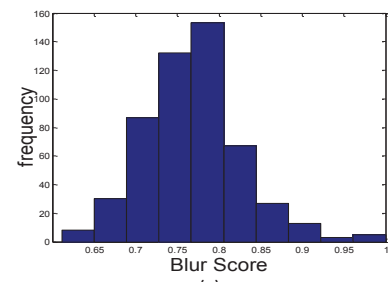

(c)

Figure 14. Blur Distribution of (a) UBIRIS, (b) IC and (c) CASIA Databases respectively

\section{Overall Quality Distribution}

For prediction of performance of the iris recognition system a unique quality score is desirable. A fusion technique based on PCA is employed as explained in section 4 to obtain the weights. In table 1 the weight of each quality parameter is illustrated.

The overall quality distribution for CASIA, UBIRIS and IC databases are illustrated in Fig. 15 CASIA has the highest quality distribution, followed by IC and then UBIRIS.

IC suffers quality degrading with respect to sharpness, dilation and blur, which is visually evident. These parameters have high weight on the quality score of IC which results in low quality. The reason for this problem is the mere fact that the iris capturing session for this database was done in an environment with light which caused reflections, resulting in the images being less clear. Moreover, individuals were required to focus their eyes to a mirror for a certain period which caused their pupil to dilate. Also, the camera captured iris images automatically and required individuals to be still which caused some discomfort and as the individual became tired and moved, which resulted in the camera capturing blurred images.

As stated in Section 6 iris images for the UBIRIS database were captured in an environment that introduced noisy images affected by diverse problems with respect to reflection, contrast, fo- cus and luminosity. The results of individual parameters also indicates that this database is affected by sharpness, dilation, area ratio and blur which is caused by the environment condition. That is why there are more low quality scores for this database.

When grading these data sets in terms of quality scores obtained on the plots, CASIA scores the highest, followed by IC and then UBIRIS.
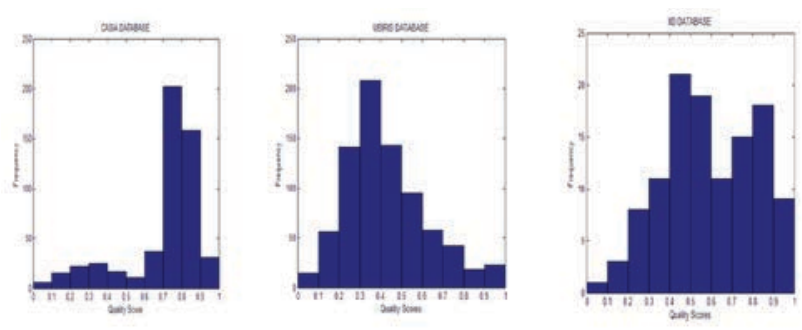

Figure 15. Overall Quality Distribution of CASIA, UBIRIS and IC Databases

\section{Conclusion}

The main aim of this research was to propose a novel iris quality assessment for an online biometric system based on quality components. Moreover,the research aimed to assess the effect of quality character components on online biometric systems.

The proposed assessment algorithm was assessed using the collected database- IC, and publicly available databases CASIA and UBIRIS.

To evaluate iris sample quality, seven quality parameters, namely, entropy, blur, contrast, area ratio, dilation, sharpness and occlusion were assessed individually and combined to form a quality score. From the quality parameters defined, a conclusion has been drawn that the combination of blur, dilation and sharpness parameters have the most influence in the quality of the image.

The proposed algorithm is open for new iris quality parameters that may emerge through future research or through further sources of acquisition constraints e.g. distance where the iris image is captured, uniform lighting. Development of a robust segmentation that will correctly localize the iris image may improve the performance of iris quality assessment metrics. 
Table 1. The weight each parameter has on quality score for each database

\begin{tabular}{|c|c|c|c|c|c|c|c|}
\hline DATABASE & $M_{C}$ & $M_{E}$ & $M_{O}$ & $M_{S}$ & $M_{A}$ & $M_{D}$ & $M_{B}$ \\
\hline CASIA & -0.3426 & 0.4235 & -0.4953 & -0.3159 & 0.5066 & 0.4122 & 0.1481 \\
\hline UBIRIS & 0.5766 & 0.4608 & -0.3099 & -0.5 & -0.16666 & -0.3764 & -0.1577 \\
\hline IC & 0.5050 & 0.5747 & 0.4028 & -0.4073 & -0.2051 & -0.0392 & -0.0780 \\
\hline
\end{tabular}

\section{References}

[1] Gulmire, K. and Ganorkar,S., Iris recognition using Gabor wavelet., International Journal of Engineering, 1(5), 2012.

[2] Masek, L.: Recognition of human iris patterns for biometric identification. $\mathrm{PhD}$ thesis.

[3] Ma, L., Tan, T., Wang, Y. and Zhang, D.,:Personal identification based on iris texture analysis., Pattern Analysis and Machine Intelligence, IEEE Transactions on, 25(12):1519- 1533, 2003.

[4] Daugman, J.:How iris recognition works., Circuits and Systems for Video Technology, IEEE Transactions on, 14(1):21-30, 2004.

[5] Fatukasi,O., Kittler, J., and Poh, N., :Quality controlled multi-modal fusion of biometric experts., In Progress in Pattern Recognition, Image Analysis and Applications, pages 881-890. Springer, 2007.

[6] Kalka,N. D., Dorairaj, V.,Shah,Y. N., Schmid, N. A. and Cukic B.,: Image quality assessment for iris biometric., In Proceedings of the 24th Annual Meeting of the Gesellscha it Klassikation, pages 445-452. Springer, 2002.

[7] Sandre, S-L and Stevens, M. and Mappes, J.,: The effect of predator appetite, prey warning coloration and luminance on predator foraging decisions, Behaviour, vol.147, No. 9., 1121-1143, BRILL, 2010

[8] Du, Y. and Belcher, C. and Zhou, Z. and Ives, R.,: Feature correlation evaluation approach for iris feature quality measure, Signal processing, Vol. 90, No. 4, 1176-1187, Elsevier, 2010

[9] Nill, N. B, IQF (Image Quality of Fingerprint) Software Application, The MITRE Corporation, 2007

[10] Crete, F., Dolmiere,T., Ladret, P. and Nicolas, M.: The blur effect: perception and estimation with a new no-reference perceptual blur metric., Human Vision and Electronic Image in XII, 6492:64920I, 2007.

[11] Li, Y.H., Savvides, M.: An automatic iris occlusion estimation method based on high-dimensional density estimation., Pattern Analysis Machine Intelligence,IEEE Transactions on, pp 784-9-6,35(4), 2013.

[12] Yalamanchili, R. K.: Occlussion Metrics, West virginia University, 2011

[13] Bieroza, M. and Baker, A. and Bridgeman, J.,: Classification and calibration of organic matter fluorescence data with multiway analysis methods and artificial neural networks: an operational tool for improved drinking water treatment, Environmetrics, Vol. 22, No.3, 256-270, Wiley Online Library, 2011

[14] Jeong, D. H. and Ziemkiewicz, C. and Ribarsky, W. and Chang, R. and Center, C. V.,:Understanding Principal Component Analysis Using a Visual Analytics Tool, Charlotte Visualization Center, UNC Charlotte, 2009

[15] Suhr, D. D.:Principal component analysis vs. exploratory factor analysis, SUGI 30 Proceedings, 203-230, 2005

[16] Proença, H. and Alexandre, L.A., UBIRIS: A noisy iris image database, International Conference on Image Analysis and Processing, 2005

[17] Chinese Academy of Sciences Institute of Automation., CASIA Iris Database, Online: http://biometrics.idealtest.org/dbDetailForUser .do?id=4, 2012

[18] Fairchild M, D:Color Appearance Models, Slides from a tutorial at the IST/SID 12th Color Imaging Conference, 2004. 